\title{
CORRELAÇÃO E VARIABILIDADE ESPACIAL DE ATRIBUTOS QUÍMICOS DO SOLO E PRODUÇÃO DE BANANEIRA 'PRATA-ANÃ'1
}

\author{
MOISES ZUCOLOTO², JULIÃO SOARES DE SOUZA LIMA ${ }^{3}$ RUIMARIO INACIO COELHO ${ }^{4}$
}

Resumo - O objetivo foi de avaliar a variabilidade espacial de atributos químicos do solo e da produção de bananeira 'Prata-Anã', por meio da estatística clássica e da geoestatística e a correlação entre eles. Foram coletadas quatro amostras ao redor de cada planta e feita uma amostra composta, na profundidade de 0-0,2 $\mathrm{m}$, em malha regular, totalizando 100 pontos amostrais espaçados de 6 x $4 \mathrm{~m}$. A produção da bananeira apresentou correlação negativa de $0,20 \mathrm{com} \mathrm{H}+\mathrm{Al}$. Todos os atributos químicos do solo e a produção da bananeira apresentaram dependência espacial.

Palavras chaves: Musa spp., geoestatística, krigagem.

\section{SPATIAL VARIABILITY OF CHEMICAL ATTRIBUTES IN THE SOIL AND YIELD OF BANANA 'PRATA AN $\tilde{A}$ '}

\begin{abstract}
The aim was to study the spatial variability of the soil chemical properties and the production of banana 'Prata-Anã', by classical statistics and geostatistics, and the correlation between them. Samples were collected at four points around the plants making a composite sample, at a depth of 0-0.2 $\mathrm{m}$ in a regular grid, total of 100 sampling points, spaced $6 \times 4 \mathrm{~m}$. The production of banana showed a negative correlation of 0.20 with $\mathrm{H}+\mathrm{Al}$. All soil chemical properties and the production of banana showed spatial dependence.
\end{abstract}

Key word: Musa spp., geostatistic, kriging.

\section{INTRODUÇÃO}

A bananeira é uma cultura produzida no Estado do Espírito Santo, ocupando uma área de 20,8 mil ha, com uma produção de 186,4 mil toneladas no ano de 2008 (IBGE, 2011), sendo uma alternativa na formação e geração de empregos diretos e indiretos, contribuindo com a diversificação da economia do Estado, da mesma forma que vem acontecendo no Estado de São Paulo (TEIXEIRA; NETO, 2011). Na produção dessa cultura, utiliza-se tecnologia especializada, abrindo, assim, espaço para a aplicação das técnicas de agricultura de precisão.

De forma geral, a variabilidade dos atributos químicos e físicos do solo influencia na qualidade e quantidade dos produtos agrícolas, indicando alta correlação entre eles. Esses fatores químicos interferem principalmente na disponibilidade de nutrientes e no condicionamento para o crescimento ideal das plantas por meio do ganho de eficiência dos fertilizantes (TEIXEIRA, et al., 2011).

Para a aplicação do manejo localizado, neces- sita-se do conhecimento do mapa da produtividade da cultura, que deve ser o primeiro passo a ser realizado, pois permite a visualização da variabilidade da produção. A correlação entre o mapa da produção e os elementos químicos permite a tomada de decisões sobre o manejo a ser adotado (BIFFI; RAFAELI, 2008).

Além de auxiliar na tomada de decisão do manejo, conhecer e quantificar a variação dos atributos de solo e os relativos à planta, é importante para auxiliar no procedimento de amostragem. Em trabalho realizado em área produtora de pimenta-do-reino (LIMA et al., 2007), o autor verificou, por meio de técnicas da geoestatística, que a variabilidade dos atributos de solo e os relativos às plantas apresentam variabilidade e dependência espacial.

Diante do exposto, objetivou-se avaliar a correlação e a variabilidade espacial de atributos químicos do solo e a produção de uma área cultivada com bananeira 'Prata-Anã', utilizando métodos de estatística clássica e geoestatística.

\footnotetext{
${ }^{1}$ Trabalho Sinfruit 112 - Simpósio Internacional de Fruticultura - Avanços na Fruticultura (17 a 21 Outubro)

Parte da Dissertação do Primeiro autor, sendo o projeto financiado pela FAPES.

${ }^{2}$ Eng. Agr., Doutorando em Fitotecnia, Câmpus Universitário, s/n, CEP: 36570000 - Viçosa-MG. E-mail: moises.zucoloto@ufv.br ${ }^{3}$ Eng. Arg., Prof. Dr. Associado II. CCA-UFES, CP 16, CEP 29500-000 - Alegre-ES. E-mail: limajss@yahoo.com.br

${ }^{4}$ Eng. Arg., Prof. Dr. Associado II. CCA-UFES, CP 16, CEP 29500-000 - Alegre-ES. E-mail: ruimario@cca.ufes.br
} 


\section{MATERIAL E MÉTODOS}

O trabalho foi conduzido em um pomar comercial localizado no Estado do Espírito Santo, cujas coordenadas geográficas são: $19^{\circ} 49^{\prime} 24^{\prime \prime}$ de latitude sul e $40^{\circ} 04^{\prime} 20^{\prime \prime}$ de longitude oeste de Greenwich. A altitude média da área de plantio está em torno de $30 \mathrm{~m}$, em declividade plana, menor que $1 \%$. O clima, segundo a classificação de Köeppen é do tipo Aw, com estação seca no inverno e verão quente e chuvoso, com temperatura média anual de $24,8^{\circ} \mathrm{C}$ e precipitação média anual acumulada de $1.200 \mathrm{~mm}$. O pomar foi instalado nos depósitos dos Tabuleiros Costeiros, e o solo, classificado como Argissolo Amarelo Distrocoeso arênico, de acordo a Embrapa (2006). A bananeira foi cultivada no espaçamento de $3 \times 2 \mathrm{~m}$, em sistema de fileira simples com manejo hídrico de irrigação por aspersão subcopa, com lâmina d'água média variando de $100 \mathrm{~mm} / \mathrm{mês}$ para os meses mais quentes a $70 \mathrm{~mm} / \mathrm{mês}$ para os meses com menor evapotranspiração.

A recomendação da adubação foi feita por meio da interpretação da fertilidade do solo para o Estado do Espírito Santo, de acordo com Prezotti et al. (2007), sendo os valores de potássio e fósforo considerados médios; e para o nitrogênio, realizou-se a recomendação para produção estimada de 30 toneladas por hectare. Ao longo do ciclo da cultura, foram aplicados $220 \mathrm{~g}$ de nitrogênio, $290 \mathrm{~g}$ de potássio e $100 \mathrm{~g}$ de fósforo, cujas fontes foram ureia, cloreto de potássio e superfosfato simples, respectivamente. A adubação foi dividida em cinco parcelas iguais e aplicada em cada touceira. Para suplementar os micronutrientes, foram realizadas duas aplicações foliares com Corona Master $^{\circledR}\left(8 \% \mathrm{~N} ; 27 \% \mathrm{P}_{2} \mathrm{O}_{5}\right.$; $0,9 \% \mathrm{Mg} ; 8 \% \mathrm{~S} ; 5 \% \mathrm{Zn} ; 0,5 \% \mathrm{H}_{3} \mathrm{BO}_{3} ; 0,1 \% \mathrm{Mo}$; $6 \% \mathrm{Mn} ; 0,1 \% \mathrm{Fe}$ e $1 \% \mathrm{Cu}$ ), na base de $4 \mathrm{~kg} \mathrm{ha}^{-1}$.

No centro da área, foi demarcada uma malha amostral regular de $2.400 \mathrm{~m}^{2}$, sendo os pontos coletados a cada $4 \mathrm{~m}$ entre plantas e $6 \mathrm{~m}$ entre linhas, totalizando 100 unidades amostrais.

As amostras de solo foram coletadas nas quatro direções, a 0,50 $\mathrm{m}$ da base das plantas (denominadas de planta-mãe), com o auxílio de uma sonda inoxidável na profundidade de 0-0,2 m, homogeneizadas, formando-se uma amostra composta. Em seguida, foram encaminhadas ao laboratório de química do solo, do CCA-UFES, para a determinação dos atributos: $\mathrm{pH}, \mathrm{P}, \mathrm{K}, \mathrm{Ca}, \mathrm{Mg}, \mathrm{Al}, \mathrm{H}+\mathrm{Al}, \mathrm{T}, \mathrm{t}, \mathrm{V}$ e m, conforme metodologia preconizada pela Embrapa (1999).

A produção da cultura foi avaliada por meio da massa do cacho por planta (MC).

A análise descritiva foi realizada determinando-se: média, mediana, valor máximo e mínimo, desvio-padrão, coeficientes de variação, assimetria e curtose. A hipótese de normalidade foi analisada pelo teste de Kolmogorov-Smirnov (KS) ( $\mathrm{p} \leq 0,05)$. Na sequência, realizou-se a análise de correlação de Pearson $(p \leq 0,05)$ entre os atributos químicos e a MC $\left(\mathrm{kg} \mathrm{planta}^{-1}\right)$.

Assumida a hipótese de estacionaridade, os dados foram submetidos à análise geoestatística por meio do ajuste do semivariograma, segundo a Equação 1.

$$
\hat{\gamma}(h)=\frac{1}{2 N(h)} \sum_{i=1}^{N(h)}\left[Z\left(x_{i}\right)-Z\left(x_{i}+h\right)\right]^{2}
$$

em que: $\mathrm{N}(\mathrm{h})$ é o número de pares de valores medidos $\mathrm{Z}\left(\mathrm{x}_{\mathrm{i}}\right), \mathrm{Z}\left(\mathrm{x}_{\mathrm{i}}+\mathrm{h}\right)$, separados por um vetor $\mathrm{h}$; e $\mathrm{Z}\left(\mathrm{x}_{\mathrm{i}}\right)$ é a variável aleatória em estudo na i-ésima posição.

O software $\mathrm{GS}^{+}$utiliza a metodologia dos mínimos quadrados para os ajustes dos modelos teóricos aos semivariogramas experimentais, determinando os parâmetros efeito pepita $\left(\mathrm{C}_{0}\right)$, patamar $\left(\mathrm{C}_{0}+\mathrm{C}\right)$ e alcance de dependência espacial (a). Os critérios para ajuste e seleção do melhor modelo foram: os coeficientes de determinação $\left(\mathrm{R}^{2}\right)$, a soma de quadrados de resíduos (SQR) e os coeficientes de correlação da validação cruzada.

Comprovada a dependência espacial dos atributos estudados, utilizou-se o método de krigagem ordinária para a interpolação dos valores dos atributos com auxílio do software Surfer.

\section{RESULTADOS E DISCUSSÃO}

Por meio da análise descritiva exposta na Tabela 1 , verifica-se distribuição assimétrica à direita para $\mathrm{MC}, \mathrm{pH}, \mathrm{P}, \mathrm{K}, \mathrm{Mg}, \mathrm{Al}$, T e m, indicando maior concentração dos dados abaixo da média e os demais com assimetria à esquerda. Em relação ao coeficiente de curtose, que é o grau de achatamento da curva de distribuição, os atributos $\mathrm{P}, \mathrm{K}, \mathrm{Mg}$ e $\mathrm{Al}$ apresentaram distribuição leptocúrtica, e os restantes apresentaram distribuição platicúrtica. Por meio do teste de Kolmogorov-Smirnov com exceção do $\mathrm{P}, \mathrm{Al}, \mathrm{H}+\mathrm{Al}$ e $\mathrm{m}$, os demais atributos apresentaram distribuição normal, fato que se verifica com a observação da proximidade da média em relação à mediana.

A MC apresenta baixa correlação negativa com o $\mathrm{H}+\mathrm{Al}$ (Tabela 2), demonstrando que o alumínio, juntamente como o hidrogênio presente no solo são prejudiciais à produção.

De acordo com a classificação agronômica para interpretação da fertilidade do solo para o Estado do Espírito Santo, os valores obtidos são classificados como médios para o $\mathrm{P}, \mathrm{K}, \mathrm{Mg}, \mathrm{Al}, \mathrm{H}+\mathrm{Al}$, T e V, e 
baixos para os demais, $\mathrm{pH}, \mathrm{Ca}, \mathrm{S} . \mathrm{B} ., \mathrm{t}$ e $\mathrm{m}$ (PREZOTTI et al., 2007). Devido à variabilidade quanto aos níveis dos elementos, recomenda-se o manejo diferenciado para suprir a provável deficiência e, consequentemente, perda de produção.

Confirmada a não violação da hipótese intrínseca, necessária para o emprego da geoestatística, por meio do estudo da estacionaridade dos dados, construíram-se os semivariogramas pela variância dos dados na análise da dependência espacial, com os dados dos parâmetros e modelos teóricos ajustados aos dados (Tabela 3 ).

Os valores de alcance relativo aos semivariogramas têm importância considerável na determinação do limite da dependência espacial, podendo ser, também, um indicativo do intervalo entre unidades de mapeamento do solo (GRECO; VIEIRA, 2005). $\mathrm{O} \mathrm{T}$ foi o atributo que apresentou maior dependência espacial (46 m), e o K, a menor (10 m); mas, de maneira geral, os atributos apresentaram alcances baixos comparados com os obtidos por Silva et al. (2008) para a cultura do café. O alcance indica a independência das amostras, pois quanto menor o alcance, mais rapidamente é obtida a independência, uma vez que o alcance é a distancia limite da dependência espacial.

Para os atributos que apresentaram dependência espacial, construíram-se mapas temáticos por krigagem ordinária com o intuito de estimar valores em locais não medidos (Figuras 1; 2; 3 e 4). Os mapas de atributos químicos apresentaram grande variabilidade na área. Observa-se uma grande amplitude nos atributos químicos estudados, ao qual podem ocorrer problemas quando se usa a média dos valores para o manejo da fertilidade. Em alguns casos, a aplicação de fertilizantes será inferior à dose necessária, em outros, a aplicação será condizente com as necessidades e, ainda, poderá haver aplicação excessiva.

Para o elemento P, que dentro dos macronutrientes é o exigido em menor quantidade (DAMATTO Jr et al., 2006), observa-se, na Figura 1, que, na maior parte da área, o nutriente encontra-se com valores inferiores. Para a manutenção da produção ou um possível aumento, mesmo o nutriente não apresentando correlação com a produção, a aplicação nas áreas com níveis baixos será necessária, merecendo atenção especial no manejo da adubação nos próximos anos agrícolas.

A produção variou de 9,5 a $12 \mathrm{~kg}$ planta $^{-1} \mathrm{em}$ grande extensão da área (Figura 5). Observa-se ainda, a não existência de similaridade com a distribuição espacial dos atributos químicos do solo, o que é comprovado por apresentar apenas baixa correlação com o $\mathrm{H}+\mathrm{Al}$ e ausência de correlação com os demais atributos.

TABELA 1 - Estatísticas descritivas dos atributos químicos do solo na profundidade de 0-0,2 m.

\begin{tabular}{|c|c|c|c|c|c|c|c|c|c|}
\hline \multirow[b]{2}{*}{ Atributos } & \multirow[b]{2}{*}{ Média } & \multirow[b]{2}{*}{$\mathrm{Md}$} & \multirow[b]{2}{*}{$\mathrm{s}$} & \multicolumn{2}{|c|}{ Valores } & \multicolumn{3}{|c|}{ Coeficientes } & \multirow{2}{*}{ DN } \\
\hline & & & & Mín & Máx & $\mathrm{CV}(\%)$ & $\mathrm{C}_{\mathrm{S}}$ & $\mathrm{C}_{\mathrm{k}}$ & \\
\hline $\mathrm{MC}(\mathrm{kg})$ & 11,05 & 11,0 & 1,99 & 6,5 & 15,50 & 17,98 & 0,25 & $-0,43$ & ns \\
\hline pH (em água) & 4,69 & 4,60 & 3,80 & 3,8 & 5,80 & 9,35 & 0,32 & $-0,47$ & ns \\
\hline $\mathrm{P}\left(\mathrm{mg} \mathrm{dm}{ }^{-3}\right)$ & 21,19 & 17,00 & 5,00 & 13,43 & 66,00 & 63,37 & 1,61 & 2,38 & $*$ \\
\hline $\mathrm{K}\left(\mathrm{mg} \mathrm{dm} \mathrm{m}^{-3}\right)$ & 58,85 & 54,00 & 17,00 & 24,91 & 138 & 42,32 & 0,99 & 0,83 & ns \\
\hline $\mathrm{Ca}\left(\mathrm{cmol}_{\mathrm{c}} \mathrm{dm}^{-3}\right)$ & 1,42 & 1,50 & 0,45 & 0,59 & 3,00 & 41,44 & $-0,13$ & $-0,62$ & $*$ \\
\hline $\operatorname{Mg}\left(\mathrm{cmol}_{\mathrm{c}} \mathrm{dm}^{-3}\right)$ & 0,83 & 0,80 & 0,40 & 0,29 & 1,80 & 35,61 & 0,88 & 1,37 & ns \\
\hline $\mathrm{Al}\left(\mathrm{cmol}_{\mathrm{c}} \mathrm{dm}^{-3}\right)$ & 0,50 & 0,40 & 0,00 & 0,52 & 4,00 & 103,79 & 3,29 & 19,92 & $*$ \\
\hline $\mathrm{H}+\mathrm{Al}\left(\mathrm{cmol}_{\mathrm{c}} \mathrm{dm}^{-3}\right)$ & 4,91 & 5,25 & 2,90 & 1,04 & 6,80 & 21,21 & $-0,12$ & $-1,34$ & $*$ \\
\hline S.B. $\left(\mathrm{cmol}_{\mathrm{c}} \mathrm{dm}^{-3}\right)$ & 2,48 & 2,60 & 1,04 & 0,88 & 5,14 & 35,44 & 0,31 & $-0,20$ & ns \\
\hline $\mathrm{T}\left(\mathrm{cmol}_{\mathrm{c}} \mathrm{dm}^{-3}\right)$ & 7,32 & 7,16 & 5,40 & 0,99 & 9,84 & 13,50 & 0,40 & $-0,70$ & ns \\
\hline $\mathrm{t}\left(\mathrm{cmol}_{\mathrm{c}} \mathrm{dm}^{-3}\right)$ & 2,87 & 2,97 & 1,45 & 0,59 & 4,43 & 20,50 & $-0,09$ & $-0,16$ & ns \\
\hline V $(\%)$ & 33,51 & 33,70 & 15,85 & 10,75 & 55,03 & 32,08 & 0,00 & $-1,19$ & ns \\
\hline $\mathrm{m}(\%)$ & 17,96 & 12,45 & 0,00 & 15,94 & 65,22 & 88,77 & 0,71 & $-0,45$ & $*$ \\
\hline
\end{tabular}

MC: massa do cacho - $\mathrm{kg}_{\text {planta }}{ }^{-1}$, Md: mediana, s: desvio-padrão, Mín: valor mínimo, Máx: valor máximo, CV: coeficiente de variação, Cs: coeficiente de assimetria, $\mathrm{C}_{\mathrm{K}}$ : coeficiente de curtose, DN: teste da distribuição normal, ns: não significativo a $5 \%$, pelo teste de Kolmogorov-Smirnov (KS), portanto distribuição normal dos dados e *: distribuição não normal. 
TABELA 2 - Coeficientes de correlação de Pearson entre os atributos químicos do solo e a produção da bananeira.

\begin{tabular}{lccccccccc}
\hline Atributos & $\mathrm{Ca}$ & $\mathrm{Mg}$ & $\mathrm{Al}$ & $\mathrm{H}+\mathrm{Al}$ & $\mathrm{SB}$ & $\mathrm{T}$ & $\mathrm{t}$ & $\mathrm{V}$ & $\mathrm{m}$ \\
\hline $\mathrm{MC}$ & & & & $-0,20^{*}$ & & & & & \\
$\mathrm{pH}$ & $0,74^{*}$ & $0,54^{*}$ & $-0,59^{*}$ & $-0,39^{*}$ & $0,69^{*}$ & & $0,30^{*}$ & $0,70^{*}$ & $-0,79^{*}$ \\
$\mathrm{P}$ & & $0,29^{*}$ & & $0,32^{*}$ & & $0,44^{*}$ & & & \\
$\mathrm{Ca}$ & & $0,76^{*}$ & $-0,51^{*}$ & $-0,37^{*}$ & $0,95^{*}$ & $0,39^{*}$ & $0,73^{*}$ & $0,87^{*}$ & $-0,79^{*}$ \\
$\mathrm{Mg}$ & & & $-0,31^{*}$ & $-0,20^{*}$ & $0,91^{*}$ & $0,52^{*}$ & $0,80^{*}$ & $0,73^{*}$ & $-0,59^{*}$ \\
$\mathrm{Al}$ & & & & $0,28^{*}$ & $-0,47^{*}$ & & & $-0,49^{*}$ & $0,86^{*}$ \\
$\mathrm{H}+\mathrm{Al}$ & & & & & $-0,34^{*}$ & $-0,68^{*}$ & & $-0,73^{*}$ & $0,36^{*}$ \\
$\mathrm{SB}$ & & & & & & $0,46^{*}$ & $0,80^{*}$ & $0,88^{*}$ & $-0,76^{*}$ \\
$\mathrm{~T}$ & & & & & & $0,45^{*}$ & & $-0,26^{*}$ \\
$\mathrm{t}$ & & & & & & & $0,66^{*}$ & $-0,28^{*}$ \\
$\mathrm{~V}$ & & & & & & & & & $-0,75^{*}$ \\
\hline
\end{tabular}

*significativo ao nível de 5\% de probabilidade; MC: massa do cacho $\left(\mathrm{kg} \mathrm{planta}^{-1}\right)$.

TABELA 3- Parâmetros e modelos dos semivariogramas utilizados.

\begin{tabular}{lcccccccc}
\hline \multirow{2}{*}{ Atributos } & Modelo & $\mathrm{a}(\mathrm{m})$ & $\mathrm{C}_{0}$ & $\mathrm{C}_{0}+\mathrm{C}$ & $\mathrm{IDE}(\%)$ & $\mathrm{R}^{2}(\%)$ & \multicolumn{2}{c}{ Validação cruzada } \\
\cline { 7 - 9 } & & & & & & & $\mathrm{R}$ & $\mathrm{p}$-valor \\
\hline $\mathrm{MC}$ & $\mathrm{EXP}$ & 11 & 0,16 & 0,95 & 95 & 59 & 37 & 0,000 \\
$\mathrm{pH}$ & $\mathrm{EXP}$ & 21 & 0,16 & 1,11 & 86 & 93 & 47 & 0,000 \\
$\mathrm{P}$ & $\mathrm{EXP}$ & 26 & 0,56 & 1,11 & 50 & 93 & 20 & 0,006 \\
$\mathrm{~K}$ & $\mathrm{ESF}$ & 10 & 0,15 & 1,00 & 85 & 59 & 30 & 0,000 \\
$\mathrm{Ca}$ & $\mathrm{EXP}$ & 22 & 0,10 & 1,06 & 90 & 78 & 67 & 0,000 \\
$\mathrm{Mg}$ & $\mathrm{ESF}$ & 36 & 0,46 & 1,24 & 61 & 97 & 55 & 0,000 \\
$\mathrm{Al}$ & $\mathrm{ESF}$ & 16 & 0,31 & 1,08 & 73 & 93 & 58 & 0,000 \\
$\mathrm{H}+\mathrm{Al}$ & $\mathrm{ESF}$ & 39 & 0,09 & 1,30 & 93 & 97 & 80 & 0,000 \\
$\mathrm{SB}$ & $\mathrm{ESF}$ & 38 & 0,09 & 1,28 & 93 & 98 & 68 & 0,000 \\
$\mathrm{~T}$ & $\mathrm{EXP}$ & 46 & 0,08 & 1,18 & 93 & 92 & 72 & 0,000 \\
$\mathrm{t}$ & $\mathrm{EXP}$ & 45 & 0,27 & 1,30 & 79 & 93 & 57 & 0,000 \\
$\mathrm{~V}$ & $\mathrm{ESF}$ & 33 & 0,20 & 1,20 & 83 & 92 & 75 & 0,000 \\
$\mathrm{~m}$ & $\mathrm{GAU}$ & 18 & 0,47 & 1,07 & 56 & 81 & 61 & 0,000 \\
\hline
\end{tabular}

MC: massa do cacho (kg planta $\left.{ }^{-1}\right)$; ESF: modelo esférico; EXP: modelo exponencial; GAU: modelo gaussiano; a: alcance; $\mathrm{C}_{0}$ : efeito pepita; $\mathrm{C}_{0}+\mathrm{C}$ : patamar; IDE: índice de dependência espacial $\left(\mathrm{C} / \mathrm{C}_{0}+\mathrm{C}\right) ; \mathrm{R}^{2}$ : coeficiente de determinação do ajuste; R: coeficiente de correlação da validação cruzada e p-valor: nível de significância do valor observado pelo valor estimado pela validação cruzada.
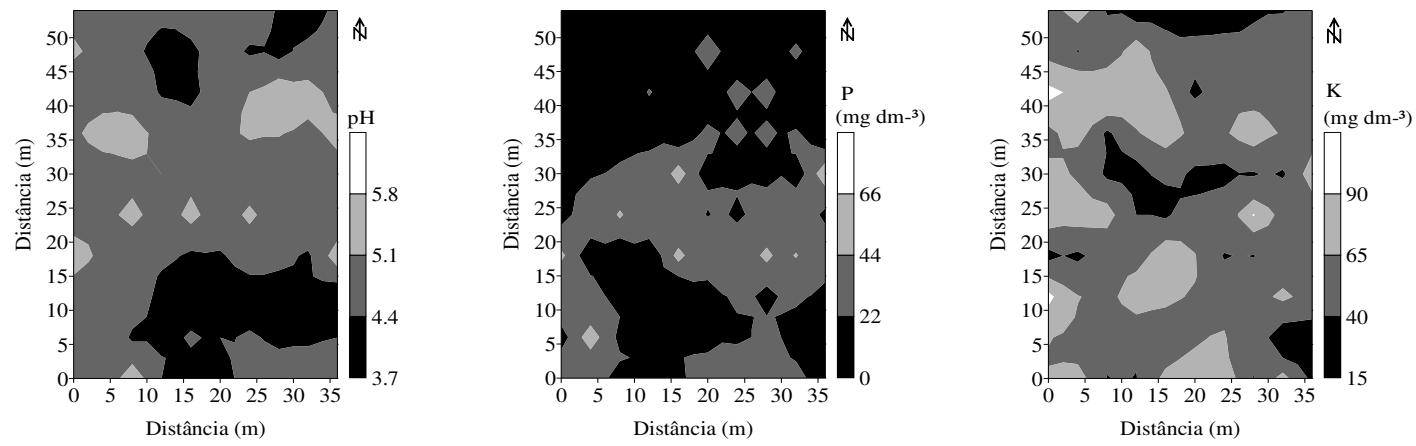

FIGURA 1- Mapas temáticos da distribuição espacial dos atributos pH, $\mathrm{P}$ e K do solo. 

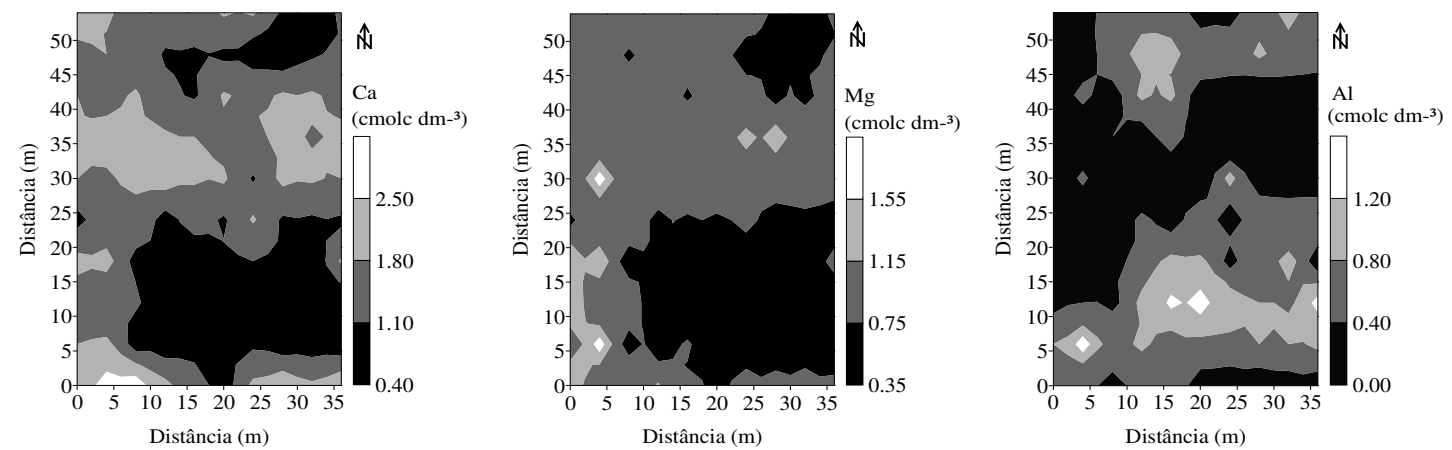

FIGURA 2- Mapas temáticos da distribuição espacial dos atributos $\mathrm{Ca}, \mathrm{Mg}$ e $\mathrm{Al}$ do solo.
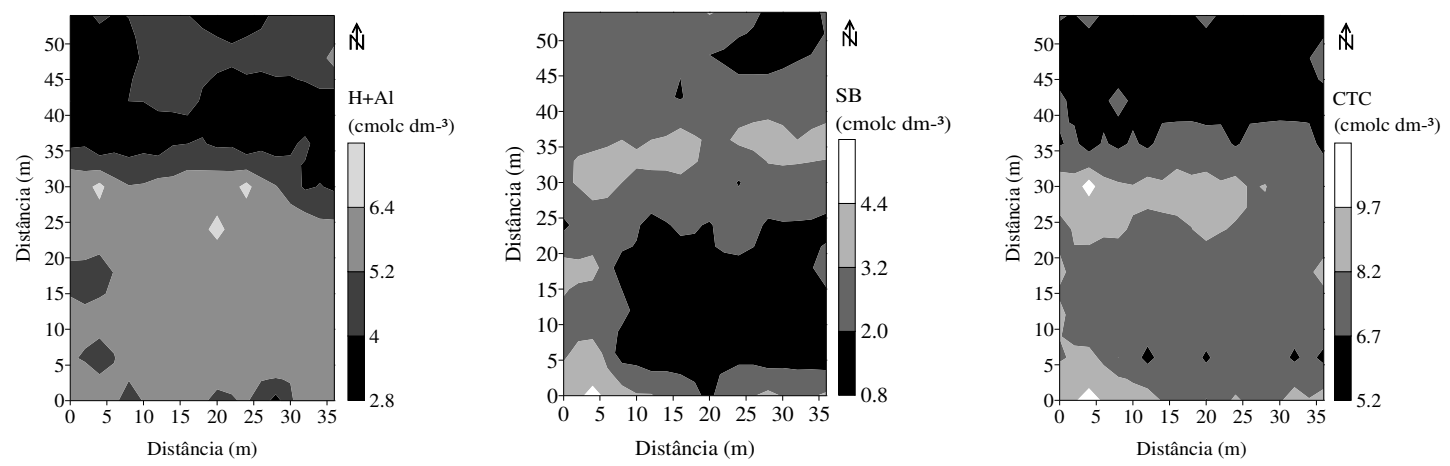

FIGURA 3- Mapas temáticos da distribuição espacial dos atributos $\mathrm{H}+\mathrm{Al}$, $\mathrm{SB}$ e $\mathrm{T}$ do solo.
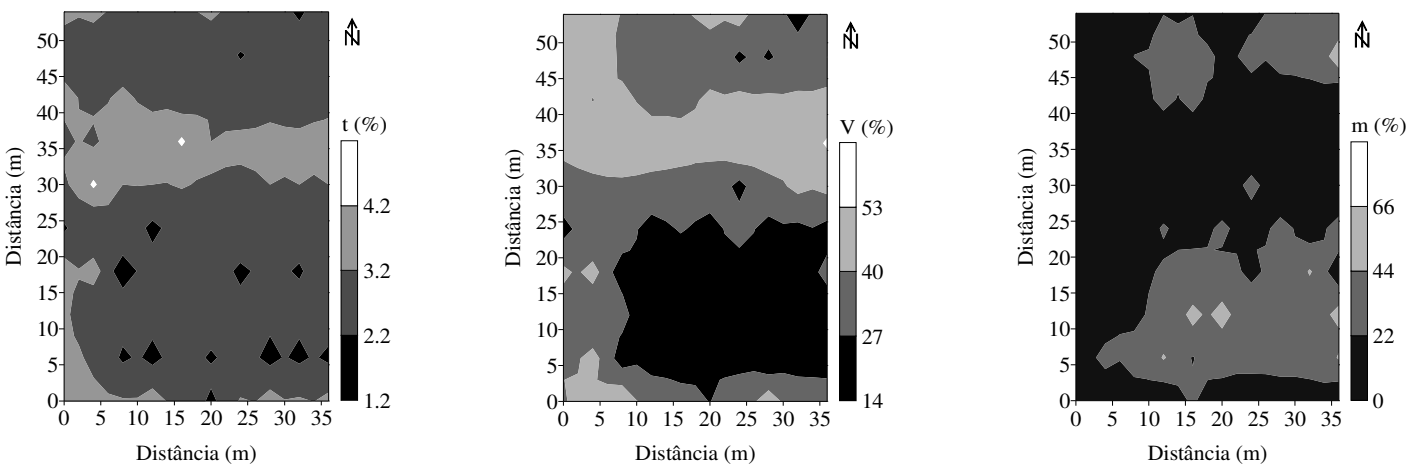

FIGURA 4 - Mapas temáticos da distribuição espacial dos atributos t, V e m do solo.

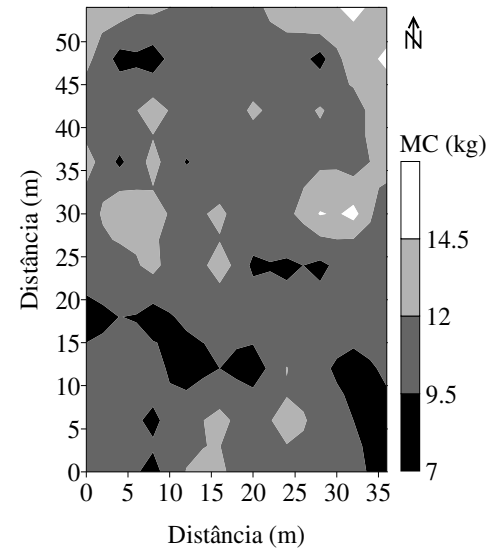

FIGURA 5 - Mapa temático da distribuição espacial da produção de banana (massa do cacho por planta$\mathrm{kg} /$ planta). 


\section{CONCLUSÕES}

1- A produção da bananeira apresentou correlação negativa de $0,20 \mathrm{com}$ o $\mathrm{H}+\mathrm{Al}$.

2- Todos os atributos químicos do solo e a produção da bananeira apresentaram dependência espacial.

\section{AGRADECIMENTO}

À pessoa de Bonfilho Zucoloto, pelo fornecimento da área para a execução do experimento.

\section{REFERÊNCIAS}

BIFFI, L.J.; RAFAELI NETO, S. L. Comportamento espacial de variáveis agronômicas da maçã 'Fuji' durante dois anos de observações no planalto serrano de Santa Catarina. Revista Brasileira de Fruticultura, Jaboticabal, v.30, n.4, p.975-980, 2008.

DAMATTO Jr, E.R.; BOAS, R. L.V.; LEONEL, S.; FERNADES, D.M. Avaliação nutricional em folhas de bananeira 'prata-anã' adubadas com composto orgânico. Revista Brasileira de Fruticultura, Jaboticabal, v.28, n.1, p.109-112, 2006.

EMBRAPA. Sistema Brasileiro de Classificação de Solos. 2.ed. Rio de Janeiro: Centro Nacional de Pesquisas de Solos, 2006. 306p.

EMBRAPA. Manual de métodos de análise de solo. 2.ed. Rio de Janeiro, Centro Nacional de Pesquisa de Solos, 1999. 212p.

GRECO, C.R.; VIEIRA, R.S. Variabilidade espacial de propriedades físicas do solo em uma parcela experimental. Revista Brasileira de Ciência do Solo, Viçosa, MG, v.29, n.2, p.169-177, 2005.
IBGE - INSTITUTO BRASILEIRO DE GEOGRAFIA E ESTATÍSTICA. Banana: área plantada e quantidade produzida. Espírito Santo: IBGE, 2011. Disponível em: <http:// www.ibge.gov.br $>$. Acesso em: 8 jul. 2011.

LIMA, J.S. de S.; OLIVEIRA, R.B.; QUARTEZANI, W.Z. Variabilidade espacial de atributos físicos de um Latossolo Vermelho-Amarelo sob cultivo de pimenta-do-reino. Engenharia na Agricultura, Viçosa, MG, v.15, n.3, p.290-298, 2007.

PREZOTTI, L.C.; GOMES, J.A.; DADALTO, G.G.; OLIVEIRA, J.A. de. Manual de recomendação de calagem e adubação para o Estado do Espírito Santo: $5^{a}$ aproximação. Vitória, 2007. 305p.

SILVA, F.M.; SOUZA, Z.M.; FIGUEIREDO, C.A.P.; VIEIRA, L.H.S.; OLIVEIRA, E. Variabilidade espacial de químicos e produtividade da cultura do café em duas safras agrícolas. Ciência e Agrotecnologia, Lavras, v.32, n.1, p.231-241, 2008.

TEIXEIRA, L.A.J.; NETO, J.E.B. Comportamento agronômico de bananeira 'Prata-Anã' em função do tipo da muda. Revista Brasileira de Fruticultura, Jaboticabal, v.33, n.1, p.089-095, 2011.

TEIXEIRA, L.A.J.; QUAGGIO, J.A.; MELLIS, E.V. Ganhos de eficiência fertilizante em bananeira sob irrigação e fertirrigação. Revista Brasileira de Fruticultura, Jaboticabal, v.33, n.1, p.272-278, 2011. 